\title{
Sliding Mode Control Based on Observer for a Class of State-Delayed Switched Systems with Uncertain Perturbation
}

\author{
Zhaolan He, Xue Wang, Zongwei Gao, and Jingjie Bai \\ School of Automation, Harbin University of Science and Technology, Harbin, Heilongjiang 150080, China \\ Correspondence should be addressed to Zhaolan He; wbabc_007@163.com
}

Received 10 December 2012; Revised 24 February 2013; Accepted 24 February 2013

Academic Editor: Ligang Wu

Copyright ( 2013 Zhaolan He et al. This is an open access article distributed under the Creative Commons Attribution License, which permits unrestricted use, distribution, and reproduction in any medium, provided the original work is properly cited.

\begin{abstract}
This paper is concerned with a state observer-based sliding mode control design methodology for a class of continuous-time statedelayed switched systems with unmeasurable states and nonlinear uncertainties. The advantages of the proposed scheme mainly lie in which it eliminates the need for state variables to be full accessible and parameter uncertainties to be satisfied with the matching condition. Firstly, a state observer is constructed, and a sliding surface is designed. By matrix transformation techniques, combined with Lyapunov function and sliding surface function, a sufficient condition is given to ensure asymptotic stability of the overall closed-loop systems composed of the observer dynamics and the estimation error dynamics. Then, reachability of sliding surface is investigated. At last, an illustrative numerical example is presented to prove feasibility of the proposed approaches.
\end{abstract}

\section{Introduction}

A switched system consists of a finite number of subsystems described by a class of differential or difference equations and a logical law that is used to orchestrate switching between these subsystems [1]. As increasing demand, this theory is widely developed, which means that many working systems can be modeled as switched systems, such as automated highway systems [2].

It is well known that different switching strategies produce different systems stability and performance, and the systems states cannot be directly measured. Accordingly, choosing a suitable switching law that stabilizes switched systems and designing an observer become an important problem [3]. Various methods of observer design have been successfully proposed, such as algebraic transfer function and singular-value decomposition. However, designing state observer turns out to be much more difficult when the system is nonlinear [4] and uncertain [5]. In [6], this problem of state observer is considered for discrete time delay switched systems with Lipschitz's nonlinearity and random switching law.

Since 1916, sliding mode control (SMC) has been proven to be an effective robust control strategy for hybrid or uncertain systems [7]. SMC belongs to variable structure control (VSC), which utilizes a discontinuous control to force the state trajectories of the system to some specific sliding surfaces on which the system acquires desired properties such as decay speed, disturbance rejection capability, and robustness. Developments on SMC involve uncertain systems $[8,9]$, time-delay systems $[10,11]$, fuzzy systems $[12,13]$, and Markovian jump systems [14-18].

Full state feedback is not always available and measurable in many practical systems. In Wu's work [19] a new robust stability condition based on observer is proposed for a class of uncertain nonlinear neutral delay systems by using the sliding mode control theory combined with reaching law technique. In He's paper [20], a sliding mode control strategy of uncertain switched linear systems based on observer is presented. The average dwell time is introduced and the matching condition of parameter uncertainties needs not to be satisfied. As the paper $[19,20]$ improved, this paper presents a new observer design for a class of uncertain nonlinear state-delayed switched systems by using the sliding mode control theory.

In the present paper, the motivation of our work is to introduce an SMC scheme based on observer for a class of switched systems that are uncertain nonlinear state-delayed systems with unmeasurable state, unknown nonlinear function, and mismatching parameter uncertainties. This is a new 
problem in SMC and switched systems research areas. In this work, the sliding mode observer for each subsystem is designed to estimate full state. By matrix transformation techniques, a sufficient condition is proposed to ensure Lyapunov asymptotic stability of the overall closed-loop switched system. In addition, the derived SMC law is provided to guarantee reachability of the designed sliding surface. Finally, an example is given to prove the feasibility of the proposed approaches.

\section{Problem Formulation and Preliminaries}

2.1. Switched Uncertain Nonlinear State-Delayed Systems. In this work, consider the following uncertain nonlinear statedelayed switched systems:

$$
\begin{gathered}
\dot{x}(t)=\left[A_{\sigma(t)}+\Delta A_{\sigma(t)}(t)\right] x(t) \\
+\left[A_{d \sigma(t)}+\Delta A_{d \sigma(t)}(t)\right] x(t-d) \\
+B\left[u_{\sigma(t)}(t)+f_{\sigma(t)}(x(t), t)\right], \\
y(t)=C x(t), \\
x(t)=\varphi(t), \quad t \in[-d, 0],
\end{gathered}
$$

where $x(t) \in R^{n}$ denotes the vector of continuous-time state variables; $u_{\sigma(t)}(t) \in R^{m}$ denotes the vector of control inputs; $y(t) \in R^{p}$ denotes the system outputs; $f_{\sigma(t)}(x(t), t) \in R^{m}$ denotes an unknown nonlinearity; $A_{\sigma(t)}, A_{d \sigma(t)}, B$, and $C$ are known real constant matrixes of corresponding dimension and the matrix $B$ is of full column rank for arbitrary $i \in \mathrm{I}$; $\Delta A_{\sigma(t)}(t)$ and $\Delta A_{d \sigma(t)}(t)$ are unknown time-varying system parameter uncertainties; $\sigma(t):[0, \infty) \rightarrow \mathbf{I}\{1, \ldots, N\}$ is switching signal which is assumed to be a piecewise constant function of time $t$; $k$ denotes the total number of switching modes. In this paper, the notations $t_{i_{r} \text { in }}$ and $t_{i_{r} \text { out }}$ are used to denote the time at which, for the $r$ th, the $i$ th subsystem is switched in and out, respectively, that is, $\sigma\left(t_{i_{\text {rin }}}^{+}\right)=\sigma\left(t_{i_{r} \text { out }}^{-}\right)=$ $i$. With these notations, $i$ is used to replace $\sigma(t)$ for $t_{i_{r} \text { in }} \leq$ $t<t_{i_{r} \text { out }}$. $d$ is a known constant delay; $\varphi(t)$ denotes a differentiable vector-valued initial function on $[-d, 0]$.

The following assumptions are useful for the development of our work.

Assumption 1. Each of the subsystems is completely observable.

Assumption 2. The uncertain parameters are of the form

$$
\left(\Delta A_{i}(t) \Delta A_{d i}(t)\right)=E_{i} F_{i}(t)\left(H_{i} H_{d i}\right) .
$$

$\Delta A_{i}(t)$ and $\Delta A_{d i}(t)$ are composed by $E_{i}, H_{i}, H_{d i}$, and $F_{i}(t)$, where $E_{i}, H_{i}$, and $H_{d i}$ are constant matrices and $F_{i}(t)$ is a timevarying matrix function satisfying

$$
F_{i}^{T}(t) F_{i}(t) \leq I
$$

Remark 1. Obviously, by comparison with Assumption 2, the matching condition that the following function must be satisfied is a stronger condition:

$$
\left(\Delta A_{i}(t) \quad \Delta A_{d i}(t)\right)=B\left(M_{i}(t) \quad M_{d i}(t)\right) .
$$

Assumption 3. There exists a known scalar function $\rho(t, y)$ such that the nonlinearities $f_{i}(x(t), t)$, for arbitrary $i \in \mathbf{I}$, satisfy

$$
\left\|f_{i}(x(t), t)\right\| \leq \rho_{i}(t, y)
$$

2.2. Sliding Mode Control. According to the sliding mode control theory, a sliding mode function is chosen as follows:

$$
S(x, t)=B^{T} X x(t),
$$

where $X \in R^{n \times n}$ is a positive definite matrix that will be designed.

We know that an ideal sliding mode within the stateestimate space exists if there exists a finite time $t_{s}$, such that

$$
S(t)=0, \quad \dot{S}(t)=0, \quad t \geq t_{s} \quad \text { or } \quad S^{T} \dot{S}(t)<0 .
$$

The second function of (7) will be used to prove the accessibility condition. And in a conventional SMC design, a class of equivalent control laws and some switching control terms can be chosen to undertake that the accessibility condition $S^{T} \dot{S}<0$ is satisfied.

Then, from

$$
\begin{aligned}
\dot{S}=\frac{\partial S}{\partial x} \frac{\partial x}{\partial t} & =B^{T} X \dot{x}(t) \\
=B^{T} X\left\{\left[A_{i}+\Delta A_{i}(t)\right] x(t)+\left[A_{d i}+\Delta A_{d i}(t)\right] x(t-d)\right. & \\
& \left.+B\left[u_{i}(t)+f_{i}(x(t), t)\right]\right\}
\end{aligned}
$$

we get

$$
\begin{gathered}
B^{T} X\left\{\left[A_{i}+\Delta A_{i}(t)\right] x(t)+\left[A_{d i}+\Delta A_{d i}(t)\right] x(t-d)\right. \\
\left.+B\left[u_{i}(t)+f_{i}(x(t), t)\right]\right\}=0 .
\end{gathered}
$$

The solution $u_{i}(t)$ of (7), namely, sliding mode control law, can be broken down into two parts: equivalent control law $u_{\text {eqi }}(t)$ and switching control term $u_{c i}(t)$, where the equivalent control $u_{\text {eqi }}(t)$ is designed for a class of certain systems without nonlinear disturbance and the switching control $u_{c i}(t)$ is robust control of nonlinear uncertain systems.

So, $u_{\mathrm{eq} i}(t)$ and $u_{c i}(t)$ are designed as the following functions (10) and (11), respectively:

$$
\begin{gathered}
u_{\mathrm{eq} i}(t)=-\left(B^{T} X B\right)^{-1} B^{T} X\left(A_{i} x(t)+A_{d i} x(t-d)\right), \\
u_{c i}(t)=-\left[\left(\rho_{i}(t, y)+\gamma_{1 i}\right)+\gamma_{2 i}\right] \operatorname{sgn}(S(t)),
\end{gathered}
$$

where, $\gamma_{1 i}, \gamma_{2 i}$ are real positive scalars to be specified and function (10) is the solution of the following equation:

$$
B^{T} X\left[A_{i} x(t)+A_{d i} x(t-d)+B u_{\mathrm{eq} i}(t)\right]=0 .
$$

Remark 2. It should be noticed that the sliding surface function defined in (6) does not switch with switching single (so $B$ not $B_{i}$ and $X$ not $X_{i}$ are designed). It means that there is a unique nonswitched sliding surface in order to avoid repetitive jumps of the state trajectories between sliding surfaces leading to instability and chattering. 
2.3. Some Lemmas. For further analysis, some lemmas are given that are useful for stability analysis of the sliding mode dynamics and the development of other theorems.

Lemma 3 (see [21]). Let $E, F$, and $H$ be real matrices of appropriate dimensions, with $F^{T} F \leq I$; then one has that for any $\operatorname{scalar} \varepsilon>0$,

$$
E F H+H^{T} F^{T} E^{T} \leq \varepsilon^{-1} E E^{T}+\varepsilon H^{T} H .
$$

Lemma 4 (Schur complement). Let $S=\left[\begin{array}{ll}S_{11} & S_{12} \\ S_{21} & S_{22}\end{array}\right]$ be symmetrical matrix; then the following three functions are equivalent:

$$
\begin{gathered}
S<0, \\
S_{11}<0, \quad S_{22}-S_{12}^{T} S_{11}^{-1} S_{12}<0, \\
S_{22}<0, \quad S_{11}-S_{12} S_{22}^{-1} S_{12}^{T}<0 .
\end{gathered}
$$

Lemma 5 (see [22]). Given matrixes $G \in R^{n \times n}$ and $U \in R^{n \times m}$. Assume that $U$ has full rank $m<n$ and $G=G^{T}$. Then, $\delta U U^{T}-$ $G>0$ for some scalar $\delta$ if and only if $\widetilde{U}^{T} G \widetilde{U}<0$ where $\widetilde{U}$ is any matrix whose columns from basis of the null space of $U^{T}$.

When the SMC is designed, we always suppose that all of the system states are available. But, this assumption is hardly satisfied on the practical viewpoint. Hence, in the next chapter, the sliding mode control-based observer will be designed.

\section{Observer-Based Sliding Mode Control}

In this section, a sliding mode observer is designed to provide the estimate of state vector, and then a sliding mode controller is synthesized based on state estimates. Furthermore, by applying the sliding mode control and the multiple Lyapunov function technique, a sufficient condition is given to ensure the asymptotic stability of the overall closed-loop statedelayed system. Finally, we guarantee that the sliding modes within both the state estimate space and state estimation errors pace are attained, respectively.

3.1. Sliding Mode Observer Design. The sliding mode observer to be designed, for state-delayed system (1)-(4), has the form of (6), which can be given by

$$
\begin{aligned}
\dot{\hat{x}}(t)= & A_{i} \widehat{x}(t)+A_{d i} \widehat{x}(t-d)+B u_{i}(t) \\
& +B u_{e i}(t)+L_{i}(y(t)-C \widehat{x}(t)),
\end{aligned}
$$

where $\widehat{x}(t)$ denotes the estimate of system state $x(t)$ and $L_{i} \in$ $R^{n \times p}$ is the observer feedback matrix to be computed later. $u_{e i}$ is a robust control term used to eliminate impact of nonlinear $f_{i}(x(t), t)$, given by

$$
u_{e i}(t)=\left(\rho_{i}(t, y)+\gamma_{1 i}\right) \operatorname{sgn}\left(S_{e}(t)\right) .
$$

The corresponding state-estimation error dynamics is given by

$$
\begin{aligned}
\dot{e}(t)= & \left(A_{i}-L_{i} C+\Delta A_{i}(t)\right) e(t) \\
& +\left(A_{d i}-\Delta A_{d i}(t)\right) e(t-d)+\Delta A_{i}(t) \hat{x}(t) \\
& +\Delta A_{d i}(t) \hat{x}(t-d)-B_{i}\left(u_{e i}(t)-f_{i}(x(t), t)\right) .
\end{aligned}
$$

According to the sliding mode control theory and (6), $S_{e}(e(t), t)=B^{T} X e(t)$.

In (6), it is assumed that matrix $X$ satisfies

$$
B^{T} X=N C
$$

We obtain

$$
\begin{aligned}
S_{e}(e(t), t) & =N C e(t)=N C(x(t)-\hat{x}(t)) \\
& =N(y(t)-C \hat{x}(t)) .
\end{aligned}
$$

This estimation error dynamics corresponds to a nonlinear uncertain state-delayed system, which is dependent on the observer feedback matrix $L_{i}$ and state estimates $\widehat{x}(t), \widehat{x}(t-d)$. This means that stability analysis of the error dynamics (17) is dependent on the observer dynamics. So, when designing the sliding mode observer, the overall closed-loop system composed of (15) and (17) must be considered to guarantee system stability and accessibility of both the sliding surface $S_{e}(e(t), t)=B^{T} X e(t)=N(y(t)-C \hat{x}(t))=0$ in state-estimation error space and the sliding surface $\widehat{S}(\widehat{x}(t), t)=B^{T} X \widehat{x}(t)$ $=0$ in the state estimate space. Namely, as (7), the following function should be satisfied:

$$
\widehat{S}(t)=0, \quad \dot{\hat{S}}(t)=0, \quad t \geq t_{s} .
$$

Hence, as functions (10) and (11), equivalent control law and switching control term are designed as follows, respectively:

$$
\begin{gathered}
u_{\mathrm{eq} i}(t)=-\left(B^{T} X B\right)^{-1} B^{T} X\left(A_{i} \widehat{x}(t)+A_{d i} \widehat{x}(t-d)\right), \\
u_{c i}(t)=-\left[\left(\rho_{i}(t, y)+\gamma_{1 i}\right)+\gamma_{2 i}\right] \operatorname{sgn}(\widehat{S}(t)),
\end{gathered}
$$

where $\gamma_{1 i}$ and $\gamma_{2 i}$ are positive design constants, which should be chosen suitably because approaching rate and chattering of sliding mode face can be influenced by it.

Hence, we obtain the following sliding mode controller:

$$
u_{i}(t)=u_{\mathrm{eq} i}(t)+u_{c i}(t) .
$$

3.2. Stability of Closed-Loop System with Observer. Summing up, considering the design of sliding mode observer and synthesis of sliding mode controller, a sufficient condition for asymptotic stability of the overall closed-loop system can be given as follows.

Theorem 6. If there exist matrices $Y_{i}, N, X>0, Q_{1 i}>0$, and $Q_{2 i}>0$, and scalars $\delta_{1 i}>0$, and $\delta_{2 i}>0$ satisfying 


$$
\begin{aligned}
& \left(\begin{array}{ccccc}
\Pi_{1 i} & X A_{d i}+\delta_{1 i} H_{i}^{T} H_{d i} & C^{T} Y_{i}^{T}+\delta_{1 i} H_{i}^{T} H_{i} & \delta_{1 i} H_{i}^{T} H_{d i} & X E \\
A_{d i}^{T} X+\delta_{1 i} H_{d i}^{T} H_{i} & \Pi_{2 i} & \delta_{1 i} H_{d i}^{T} H_{i} & \delta_{1 i} H_{d i}^{T} H_{d i} & 0 \\
Y_{i} C+\delta_{1 i} H_{i}^{T} H_{i} & \delta_{1 i} H_{i}^{T} H_{d i} & \Pi_{3 i} & X A_{d i}+\delta_{1 i} H_{i}^{T} H_{d i} & 0 \\
\delta_{1 i} H_{d i}^{T} H_{i} & \delta_{1 i} H_{d i}^{T} H_{d i} & A_{d i}^{T} X+\delta_{1 i} H_{d i}^{T} H_{i} & \Pi_{4 i} & 0 \\
E^{T} X & 0 & 0 & 0 & -\delta_{1 i} I
\end{array}\right) \leq 0 \\
& B^{T} X=N C,
\end{aligned}
$$

where

$$
\begin{aligned}
& \Pi_{1 i}=X A_{i}+A_{i}^{T} X-Y_{i} C-C^{T} Y_{i}^{T}+Q_{1 i}+\delta_{1 i} H_{i}^{T} H_{i}-\delta_{2 i} B B^{T}, \\
& \Pi_{2 i}=-Q_{1 i}+\delta_{1 i} H_{d i}^{T} H_{d i}-\delta_{2 i} B B^{T}, \\
& \Pi_{3 i}=A_{i}^{T} X+X A_{i}+Q_{2 i}+\delta_{1 i} H_{i}^{T} H_{i}-\delta_{2 i} B B^{T}, \\
& \Pi_{4 i}=-Q_{2 i}+\delta_{1 i} H_{d i}^{T} H_{d i}-\delta_{2 i} B B^{T}
\end{aligned}
$$

then the sliding mode control law (21)-(22) guarantees that the combined closed-loop switched system is asymptotically stable for the switching signal satisfies

$$
\sigma(t)=i=\arg \left\{\min \left(V_{i}(t)\right)\right\}, \quad i \in \mathbf{I} .
$$

Furthermore, an observer feedback matrix is given by $L_{i}=$ $X^{-1} Y_{i}$.

Proof. Firstly, using the method of matrix transformation [23] function (15) is transformed, and the transformation matrix and the associated vector $\widehat{z}(t)$ are defined as

$$
\widehat{z}(t)=\left(\begin{array}{l}
\widehat{z}_{1}(t) \\
\widehat{z}_{2}(t)
\end{array}\right)=W \widehat{x}(t),
$$

where $\widehat{z}_{1}(t) \in R^{n-m}, \widehat{z}_{2}(t) \in R^{m}, \widetilde{B}$ is an orthogonal complement of $B$, which means $\widetilde{B}^{T} B=0$. So $B$ is designed to a nonsingular matrix. It is easily known that $W^{-1}=\left(\begin{array}{lll}X^{-1} \widetilde{B} & B\end{array}\right)$ and $\widehat{z}_{2}(t)=\left(B^{T} X B\right)^{-1} \widehat{s}(t)$ due to the following function:

$$
\begin{aligned}
& \left(\begin{array}{l}
\left(\widetilde{B}^{T} X^{-1} \widetilde{B}\right)^{-1} \widetilde{B}^{T} \\
\left(B^{T} X B\right)^{-1} B^{T} X
\end{array}\right)\left(\begin{array}{ll}
X^{-1} \widetilde{B} & B
\end{array}\right) \\
& \quad=\left(\begin{array}{ll}
\left(\widetilde{B}^{T} X^{-1} \widetilde{B}\right)^{-1} \widetilde{B}^{T} X^{-1} \widetilde{B} & \left(\widetilde{B}^{T} X^{-1} \widetilde{B}\right)^{-1} \widetilde{B}^{T} B \\
\left(B^{T} X B\right)^{-1} B^{T} X X^{-1} \widetilde{B} & \left(B^{T} X B\right)^{-1} B^{T} X B
\end{array}\right) \\
& =\left(\begin{array}{ll}
I & 0 \\
0 & I
\end{array}\right)=I .
\end{aligned}
$$

Because of the sliding surface $\widehat{s}(x, t)=0$, we obtain $\widehat{z}_{2}(t)=0$. So $(n-m)$ reduced-order sliding mode dynamic system as follows:

$$
\begin{aligned}
\dot{\vec{z}}_{1}= & \left(\widetilde{B}^{T} X^{-1} \widetilde{B}\right)^{-1} \widetilde{B}^{T}\left(A_{i}-L_{i} C\right) X^{-1} \widetilde{B} \widehat{z}_{1}(t) \\
& +\left(\widetilde{B}^{T} X^{-1} \widetilde{B}\right)^{-1} \widetilde{B}^{T} A_{d i} X^{-1} \widetilde{B} \widehat{z}_{1}(t-d) \\
& +\left(\widetilde{B}^{T} X^{-1} \widetilde{B}\right)^{-1} \widetilde{B}^{T} L_{i} C X^{-1} \widetilde{B} z_{1}(t) .
\end{aligned}
$$

Similar to the previous method, function (17) is transformed as

$$
\begin{aligned}
\dot{e}_{z 1}(t)= & \left(\widetilde{B}^{T} X^{-1} \widetilde{B}\right)^{-1} \widetilde{B}^{T}\left(A_{i}-L_{i} C+\Delta A_{i}(t)\right) X^{-1} \widetilde{B} e_{z 1}(t) \\
& +\left(\widetilde{B}^{T} X^{-1} \widetilde{B}\right)^{-1} \widetilde{B}^{T} \Delta A_{i}(t) X^{-1} \widetilde{B} \widehat{v}_{1}(t) \\
& +\left(\widetilde{B}^{T} X^{-1} \widetilde{B}\right)^{-1} \widetilde{B}^{T}\left(A_{d i}+\Delta A_{d i}(t)\right) X^{-1} \widetilde{B} e_{z 1}(t-d) \\
& +\left(\widetilde{B}^{T} X^{-1} \widetilde{B}\right)^{-1} \widetilde{B}^{T} \Delta A_{d i}(t) X^{-1} \widetilde{B} \widehat{v}_{1}(t-d) .
\end{aligned}
$$

Then, the Lyapunov functional is chosen as

$$
\begin{aligned}
V_{i}(t)= & e_{z 1}^{T}(t)\left(\widetilde{B}^{T} X^{-1} \widetilde{B}\right) e_{z 1}(t) \\
& +\int_{t-d}^{t} e_{z 1}^{T}(\tau)\left(\widetilde{B}^{T} \bar{Q}_{1 i} \widetilde{B}\right) e_{z 1}(\tau) d \tau \\
& +\widehat{z}_{1}^{T}(t)\left(\widetilde{B}^{T} X^{-1} \widetilde{B}\right) \widehat{z}_{1}(t) \\
& +\int_{t-d}^{t} \widehat{z}_{1}^{T}(\tau)\left(\widetilde{B}^{T} \bar{Q}_{2 i} \widetilde{B}\right) \widehat{z}_{1}(\tau) d \tau, \\
\dot{V}_{i}(t)= & \dot{e}_{z 1}^{T}(t)\left(\widetilde{B}^{T} X^{-1} \widetilde{B}\right) e_{z 1}(t)+e_{z 1}^{T}(t)\left(\widetilde{B}^{T} X^{-1} \widetilde{B}\right) \dot{e}_{z 1}(t) \\
& +\left(e_{z 1}^{T}(t)\left(\widetilde{B}^{T} \bar{Q}_{1 i} \widetilde{B}\right) e_{z 1}(t)\right)_{t-d}^{t} \\
& +\dot{\vec{z}}_{1}^{T}(t)\left(\widetilde{B}^{T} X^{-1} \widetilde{B}\right) \widehat{z}_{1}(t)+\widehat{z}_{1}^{T}(t)\left(\widetilde{B}^{T} X^{-1} \widetilde{B}\right) \dot{\vec{z}}_{1}(t) \\
& +\left(\widehat{z}_{1}^{T}(t)\left(\widetilde{B}^{T} \bar{Q}_{2 i} \widetilde{B}\right) \widehat{z}_{1}(t)\right)_{t-d}^{t}
\end{aligned}
$$

Taking the time derivative along the state trajectories of (15) and (17), and considering (21)-(22), it follows that

$$
\dot{V}_{i}(t)=Z^{T}(t) G_{i}(t) Z(t),
$$


where

$$
\begin{aligned}
& G_{i}(t)=\left(\begin{array}{cccc}
X^{-1} \widetilde{B} & 0 & 0 & 0 \\
0 & X^{-1} \widetilde{B} & 0 & 0 \\
0 & 0 & X^{-1} \widetilde{B} & 0 \\
0 & 0 & 0 & X^{-1} \widetilde{B}
\end{array}\right)^{T}\left(G_{1 i}+G_{2 i}(t)\right) \\
& \times\left(\begin{array}{cccc}
X^{-1} \widetilde{B} & 0 & 0 & 0 \\
0 & X^{-1} \widetilde{B} & 0 & 0 \\
0 & 0 & X^{-1} \widetilde{B} & 0 \\
0 & 0 & 0 & X^{-1} \widetilde{B}
\end{array}\right) \\
& Z(t)=\left(\begin{array}{c}
e_{z 1}(t) \\
e_{z 1}(t-d) \\
\widehat{z}_{1}(t) \\
\widehat{z}_{1}(t-d)
\end{array}\right) \\
& G_{1 i}=\left(\begin{array}{cccc}
\bar{\Pi}_{1 i} & X A_{d i} & C^{T} L_{i}^{T} X & 0 \\
A_{d i}^{T} X & -Q_{1 i} & 0 & 0 \\
X L_{i} C & 0 & \bar{\Pi}_{2 i} & X A_{d i} \\
0 & 0 & A_{d i}^{T} X & -Q_{2 i}
\end{array}\right) \\
& G_{2 i}(t)=\left(\begin{array}{c}
X E \\
0 \\
0 \\
0
\end{array}\right) F_{i}(t)\left(\begin{array}{llll}
H_{i} & H_{d i} & H_{i} & H_{d i}
\end{array}\right) \\
& +\left(\left(\begin{array}{c}
X E \\
0 \\
0 \\
0
\end{array}\right) F_{i}(t)\left(\begin{array}{llll}
H_{i} & H_{d i} & H_{i} & H_{d i}
\end{array}\right)\right)^{T}, \\
& \bar{\Pi}_{1 i}=\left(A_{i}-L_{i} C\right)^{T} X+X\left(A_{i}-L_{i} C\right)+Q_{1 i} \text {, } \\
& \bar{\Pi}_{2 i}=A_{i}^{T} X+X A_{i}+Q_{2 i}, \\
& Q_{1 i}=X \bar{Q}_{1 i} X, \quad Q_{2 i}=X \bar{Q}_{2 i} X .
\end{aligned}
$$

From (32), it is easily seen that $\dot{V}_{i}(t)<0$ if $G_{i}(t)<0$ for $Z \neq 0$. So, we just prove the inequality $G_{i}(t)<0$ is satisfied.
By Lemma 3, the previous matrix inequality holds for $F_{i}(t)$ satisfying $F_{i}^{T}(t) F_{i}(t) \leq I$ if there exists a constant $\delta_{1 i}>0$ such that

$$
\begin{aligned}
G_{2 i}(t) \leq \bar{G}_{2 i}= & \delta^{-1}\left(\begin{array}{c}
X E \\
0 \\
0 \\
0
\end{array}\right)\left(\begin{array}{llll}
E^{T} X & 0 & 0 & 0
\end{array}\right) \\
& +\delta\left(\begin{array}{c}
H_{i}^{T} \\
H_{d i}^{T} \\
H_{i}^{T} \\
H_{d i}^{T}
\end{array}\right)\left(\begin{array}{llll}
H_{i} & H_{d i} & H_{i} & H_{d i}
\end{array}\right) .
\end{aligned}
$$

So, $G_{i}<0$ only if

$$
\begin{gathered}
\left(\begin{array}{cccc}
X^{-1} \widetilde{B} & 0 & 0 & 0 \\
0 & X^{-1} \widetilde{B} & 0 & 0 \\
0 & 0 & X^{-1} \widetilde{B} & 0 \\
0 & 0 & 0 & X^{-1} \widetilde{B}
\end{array}\right)^{T}\left(G_{1 i}+\bar{G}_{2 i}\right) \\
\times\left(\begin{array}{cccc}
X^{-1} \widetilde{B} & 0 & 0 & 0 \\
0 & X^{-1} \widetilde{B} & 0 & 0 \\
0 & 0 & X^{-1} \widetilde{B} & 0 \\
0 & 0 & 0 & X^{-1} \widetilde{B}
\end{array}\right)<0
\end{gathered}
$$

By Lemma 4, inequality (35) is equivalent to the following inequality (36):

$$
\begin{gathered}
\left(\begin{array}{cccc}
X^{-1} \widetilde{B} & 0 & 0 & 0 \\
0 & X^{-1} \widetilde{B} & 0 & 0 \\
0 & 0 & X^{-1} \widetilde{B} & 0 \\
0 & 0 & 0 & X^{-1} \widetilde{B}
\end{array}\right){ }^{T} \\
\times G_{3 i}\left(\begin{array}{cccc}
X^{-1} \widetilde{B} & 0 & 0 & 0 \\
0 & X^{-1} \widetilde{B} & 0 & 0 \\
0 & 0 & X^{-1} \widetilde{B} & 0 \\
0 & 0 & 0 & X^{-1} \widetilde{B}
\end{array}\right)<0
\end{gathered}
$$

where

$$
G_{3 i}=\left(\begin{array}{ccccc}
\Pi_{1 i} & X A_{d i}+\delta_{1 i} H_{i}^{T} H_{d i} & C^{T} Y_{i}^{T}+\delta_{1 i} H_{i}^{T} H_{i} & \delta_{1 i} H_{i}^{T} H_{d i} & X E \\
A_{d i}^{T} X+\delta_{1 i} H_{d i}^{T} H_{i} & \Pi_{2 i} & \delta_{1 i} H_{d i}^{T} H_{i} & \delta_{1 i} H_{d i}^{T} H_{d i} & 0 \\
Y_{i} C+\delta_{1 i} H_{i}^{T} H_{i} & \delta_{1 i} H_{i}^{T} H_{d i} & \Pi_{3 i} & X A_{d i}+\delta_{1 i} H_{i}^{T} H_{d i} & 0 \\
\delta_{1 i} H_{d i}^{T} H_{i} & \delta_{1 i} H_{d i}^{T} H_{d i} & A_{d i}^{T} X+\delta_{1 i} H_{d i}^{T} H_{i} & \Pi_{4 i} & 0 \\
E^{T} X & 0 & 0 & 0 & -\delta_{1 i} I
\end{array}\right) .
$$

By Lemma 5 and given $L_{i}=X^{-1} Y_{i}$, LMI (23) is obtained. That means $\dot{V}_{i}(t)<0$ only if there exist matrices $Y_{i}, N, X>0$, $Q_{1 i}>0$, and $Q_{2 i}>0$ and scalars $\delta_{1 i}>0$, and $\delta_{2 i}>0$ satisfying LMI (23).

Remark 7. As a result of the given Theorem 6, the observerbased SMC stable problem becomes a linear matrix inequality feasibility problem. Consider the linear equality condition
$B^{T} X=N C$, where $N, X>0$ satisfies LMI (23), which can be equivalently converted to

$$
\operatorname{tr}\left(\left(B^{T} X-N C\right)^{T}\left(B^{T} X-N C\right)\right)=0 .
$$

Introduce the condition

$$
\left(B^{T} X-N C\right)^{T}\left(B^{T} X-N C\right) \leq \beta I,
$$


and Lemma 4 gives

$$
\left(\begin{array}{cc}
-\sqrt{\beta} I & X B-C^{T} N^{T} \\
B^{T} X-N C & -\sqrt{\beta} I
\end{array}\right) \leq 0 .
$$

Hence, it is now changed to a problem in which a global solution of the following minimization is found:

$\min \beta \quad$ subject to (23) and (40).

So, it is a minimization problem relating linear objective and LMI constraints; it has an infimum and when infimum equals zero, the observed-based SMC problem is solvable.

3.3. Accessibility Condition. Finally, we prove the accessibility of sliding surfaces $\widehat{S}(\widehat{x}(t), t)=0$ in the state-estimate space and $S_{e}(e(t), t)=0$ in estimation error space. So, Theorem 8 is given.

Theorem 8. If there exist matrices $Y_{i}, N, X>0, Q_{1 i}>0, Q_{2 i}>$ 0 and scalars $\delta_{1 i}>0$, and $\delta_{2 i}>0$ satisfying (23) with (24), and observer feedback matrix $L_{i}=X^{-1} Y_{i}$, then the sliding mode control law (26) with (23) and (24) guarantees that the sliding motion is attained on the sliding surfaces $\widehat{S}(\widehat{x}(t), t)=0$ and $S_{e}(e(t), t)=0$, respectively.

Proof. As (7), $\widehat{S}(\widehat{x}(t), t)=0$ and $S_{e}(e(t), t)=0$ can be replaced by $\widehat{S}^{T} \dot{\hat{S}}(t)<0$ and $S_{e}^{T} \dot{S}_{e}(t)<0$.

We know $\widehat{S}^{T} \dot{\hat{S}}(t)<0$ is the same as $\widehat{S}^{T}\left(B^{T} X B\right)^{-1} \dot{\hat{S}}(t)<$ 0 , so $\widehat{S}^{T} \dot{\hat{S}}(t)<0$ and $S_{e}^{T} \dot{S}_{e}(t)<0$ can be replaced by $\widehat{S}^{T}\left(B^{T} X B\right)^{-1} \dot{\hat{S}}(t)^{-1}<0$ and $S_{e}^{T}\left(B^{T} X B\right)^{-1} \dot{S}_{e}(t)<0$ to make proving simpler:

$$
\begin{aligned}
& \widehat{S}^{T}(t)\left(B^{T} X B\right)^{-1} \dot{\hat{S}}(t) \\
& =\widehat{S}^{T}(t)\left(B^{T} X B\right)^{-1} B^{T} X\left[A_{i} \widehat{x}(t)+A_{d i} \widehat{x}(t-d)+B u_{i}(t)\right. \\
& \left.+B u_{e i}(t)+L_{i}(y(t)-C \hat{x}(t))\right] \\
& =\widehat{S}^{T}(t)\left(B^{T} X B\right)^{-1} B^{T} X \\
& \times\left(A_{i} \hat{x}(t)+A_{d i} \widehat{x}(t-d)+L_{i} C e(t)+B u_{\mathrm{eq} i}\right) \\
& -\widehat{S}^{T}(t)\left(u_{c i}+u_{e i}\right) \\
& \leq\|\widehat{S}(t)\|\left[\left\|\left(B^{T} X B\right)^{-1}-I\right\|\right. \\
& \times\left(\left\|B^{T} X A_{i}\right\|\|\widehat{x}(t)\|+\left\|B^{T} X A_{d i}\right\|\|\widehat{x}(t-d)\|\right) \\
& \left.+\left\|\left(B^{T} X B\right)^{-1}\right\|\left\|B^{T} X L_{i} C\right\|\|e(t)\|\right] \\
& -\|\widehat{S}(t)\|\left[\left(\rho_{i}(t, y)+\gamma_{1 i}\right)+\gamma_{2 i}\right] \\
& +\|\widehat{S}(t)\|\left(\rho_{i}(t, y)+\gamma_{1 i}\right) \\
& \leq\|\widehat{S}(t)\|\left[\left(-\gamma_{2 i}+\alpha_{1 i} \alpha_{2 i}\right)\|\widehat{x}(t)\|\right. \\
& \left.+\alpha_{1 i} \alpha_{3 i}\|\widehat{x}(t-d)\|+\alpha_{4 i} \alpha_{5 i}\|e(t)\|\right],
\end{aligned}
$$

$$
\begin{aligned}
& S_{e}^{T}(t)\left(B^{T} X B\right)^{-1} \dot{S}_{e}(t) \\
& =S_{e}^{T}(t)\left(B^{T} X B\right)^{-1} B^{T} X\left[\left(A_{i}-L_{i} C+\Delta A_{i}(t)\right) e(t)\right. \\
& +\left(A_{d i}+\Delta A_{d i}(t)\right) e(t-d) \\
& -B\left(u_{e i}(t)-f_{i}(x(t), t)\right) \\
& \left.+\Delta A_{i} \widehat{x}(t)+\Delta A_{d i} \widehat{x}(t-d)\right] \\
& =-S_{e}^{T}(t)\left(u_{e i}(t)-f_{i}(x(t), t)\right) \\
& +S_{e}^{T}(t)\left(B^{T} X B\right)^{-1} B^{T} X\left[\left(A_{i}-L_{i} C+\Delta A_{i}(t)\right) e(t)\right. \\
& +\left(A_{d i}+\Delta A_{d i}(t)\right) e(t-d) \\
& \left.+\Delta A_{i} \hat{x}(t)+\Delta A_{d i} \hat{x}(t-d)\right] \\
& \leq\left\|S_{e}(t)\right\| \rho_{i}(t, y)+\left\|S_{e}(t)\right\|\left\|\left(B^{T} X B\right)^{-1}\right\| \\
& \times\left[\left\|B^{T} X\left(A_{i}-L_{i} C\right)\right\|\|e(t)\|+\left\|B^{T} X E\right\|\left\|H_{i}\right\|\|e(t)\|\right. \\
& +\left\|B^{T} X A_{d i}\right\|\|e(t-d)\|+\left\|B^{T} X E\right\|\left\|H_{d i}\right\|\|e(t-d)\| \\
& \left.+\left\|B^{T} X E\right\|\left\|H_{i}\right\|\|\widehat{x}(t)\|+\left\|B^{T} X E\right\|\left\|H_{d i}\right\|\|\widehat{x}(t-d)\|\right] \\
& \leq\left\|S_{e}(t)\right\|\left[-\gamma_{1 i}+\alpha_{4 i}\left(\beta_{1 i}-\beta_{2 i}\right)\|e(t)\|\right. \\
& +\left(\alpha_{3 i}+\beta_{3 i}\right)\|e(t-d)\|+\beta_{2 i}\|\hat{x}(t)\| \\
& \left.+\beta_{3 i}\|\widehat{x}(t-d)\|\right]
\end{aligned}
$$

with $\alpha_{1 i}=\left\|\left(B^{T} X B\right)^{-1}-I\right\|, \alpha_{2 i}=\left\|B^{T} X A_{i}\right\|, \alpha_{3 i}=\left\|B^{T} X A_{d i}\right\|$, $\alpha_{4 i}=\left\|\left(B^{T} X B\right)^{-1}\right\|, \alpha_{5 i}=\left\|B^{T} X L_{i} C\right\|, \beta_{1 i}=\| B^{T} X\left(A_{i}-\right.$ $\left.L_{i} C\right)\left\|, \beta_{2 i}=\right\| B^{T} X E\|\| H_{i}\left\|, \beta_{3 i}=\right\| B^{T} X E\|\| H_{d i} \|$.

In the state space composed of state estimate vector and estimation error vector, we define the following domain:

$$
\Omega_{\delta i}=\left\{\begin{array}{c}
Z \in R^{4 n}: \alpha_{1 i} \alpha_{2 i}\|\widehat{x}(t)\|+\alpha_{1 i} \alpha_{3 i}\|\widehat{x}(t-d)\| \\
+\alpha_{4 i} \alpha_{5 i}\|e(t)\|<\gamma_{2 i}-\delta \\
\alpha_{4 i}\left(\beta_{1 i}-\beta_{2 i}\right)\|e(t)\|+\beta_{2 i}\|\widehat{x}(t)\| \\
+\left(\alpha_{3 i}+\beta_{3 i}\right)\|e(t-d)\| \\
+\beta_{3 i}\|\widehat{x}(t-d)\|<\gamma_{1 i}-\delta
\end{array}\right\}
$$

with constant $\delta$ satisfying $0<\delta<\min \left\{\gamma_{1 i}, \gamma_{2 i}\right\}$, so from (42), in the domain $\Omega_{\delta i}$, we have $\widehat{S}^{T} \dot{\hat{S}}(t)<0$ and $S_{e}^{T} \dot{S}_{e}(t)<0$.

So, the sliding surfaces $\widehat{S}(\widehat{x}(t), t)=0$ in the state-estimate space and $S_{e}(e(t), t)=0$ in estimation error space can be accessed.

Remark 9. Only when the state trajectories enter the $\Omega_{\delta i}$, could $\widehat{S}(t)=0$ and $S_{e}(t)=0$ happen. The region in which sliding motion takes place is usually referred to as sliding patch [24]. In the present scheme, it can be seen that size of the sliding patch depends on design constants $\gamma_{1 i}, \gamma_{2 i}$. 


\section{Simulation}

In this section, the following switch system will be used to illustrate the proposed sliding mode control scheme-based observer is feasible:

$$
\begin{gathered}
\dot{x}(t)=\left[A_{\sigma(t)}+\Delta A_{\sigma(t)}(t)\right] x(t) \\
+\left[A_{d \sigma(t)}+\Delta A_{d \sigma(t)}(t)\right] x(t-d) \\
+B\left[u_{\sigma(t)}(t)+f_{\sigma(t)}(x(t), t)\right], \\
y(t)=C x(t),
\end{gathered}
$$

where

$$
\begin{aligned}
& A_{1}=\left(\begin{array}{ccc}
-2 & 4 & 0 \\
-7 & -1.5 & 0 \\
0 & 1 & -1
\end{array}\right), \quad A_{2}=\left(\begin{array}{ccc}
-1.2 & 0.9 & 0 \\
2 & -2 & 0 \\
0 & 1.4 & -1.3
\end{array}\right), \\
& A_{d 1}=\left(\begin{array}{ccc}
-0.3 & 0.1 & 0.1 \\
0.1 & 0.1 & 0 \\
0 & 0.1 & 0.2
\end{array}\right), \quad A_{d 2}=\left(\begin{array}{ccc}
0.1 & 0.2 & 0.3 \\
0.12 & 0.09 & 0 \\
0 & 0.1 & 0.2
\end{array}\right) \\
& B=\left(\begin{array}{ll}
2 & 1 \\
1 & 2 \\
1 & 1
\end{array}\right), \quad C=\left(\begin{array}{lll}
1 & 3 & 2 \\
2 & 2 & 0
\end{array}\right), \\
& E_{1}=E_{2}=\left(\begin{array}{ccc}
0.1 & 0.2 & 0.2 \\
0 & 0.2 & 0.1 \\
0 & 0 & 0.3
\end{array}\right) \\
& H_{1}=H_{2}=\left(\begin{array}{ccc}
0.1 & 0.2 & 0.1 \\
0 & 0.2 & 0.3 \\
0.2 & 0.2 & 0.1
\end{array}\right) \\
& H_{d 1}=H_{d 2}=\left(\begin{array}{ccc}
0.2 & 0 & 0.1 \\
0 & 0.1 & 0.1 \\
0.1 & 0.1 & 0.2
\end{array}\right) \\
& f_{1}(x(t), t)=\left(\begin{array}{c}
0.4 \sin \left(x_{1}(t)\right) \\
0.2 \cos \left(x_{2}(t)\right)
\end{array}\right), \\
& f_{2}(x(t), t)=\left(\begin{array}{c}
0.3 \sin \left(x_{2}(t)\right) \\
0.3 \cos \left(x_{3}(t)\right)
\end{array}\right), \\
& F_{1}(t)=\left(\begin{array}{ccc}
\sin (t) & 0 & 0 \\
0 & \sin (t) & 0 \\
0 & 0 & \cos (t)
\end{array}\right) \\
& F_{2}(t)=\left(\begin{array}{ccc}
\cos (t) & 0 & 0 \\
0 & \sin (t) & 0 \\
0 & 0 & 1
\end{array}\right) \\
& d(t)=2 \sin (t)+1, \quad d=3, \\
& x(t)=\left(\begin{array}{lll}
1 & -1 & 0
\end{array}\right)^{T}, \quad t \in[-2,0], \\
& x(0)=\left(\begin{array}{lll}
1 & -1 & 0.5
\end{array}\right)^{T} .
\end{aligned}
$$

Then, the linear inequalities (23) and (24) with (41) are solved, and we obtain:

$$
\begin{aligned}
& X=\left(\begin{array}{ccc}
2.8965 & -0.8585 & -5.1634 \\
-0.8585 & 1.7945 & 1.2445 \\
-5.1634 & 1.2445 & 10.6332
\end{array}\right) \\
& N=\left(\begin{array}{ll}
0.7754 & -0.5022 \\
3.9794 & -3.9817
\end{array}\right) \\
& Y_{1}=\left(\begin{array}{cc}
-4.3062 & 0.7478 \\
-3.3264 & 0.1157 \\
-1.9617 & -1.3973
\end{array}\right) \text {, } \\
& Y_{2}=\left(\begin{array}{cc}
-0.6094 & 0.4116 \\
0.5758 & -0.6553 \\
-0.0320 & -0.1995
\end{array}\right) \text {, } \\
& L_{1}=\left(\begin{array}{cc}
-16.3887 & 0.3210 \\
-4.4045 & 0.2188 \\
-7.6272 & -0.0012
\end{array}\right) \text {, } \\
& L_{2}=\left(\begin{array}{cc}
-1.5303 & 0.6209 \\
0.1156 & -0.2876 \\
-0.7596 & 0.3164
\end{array}\right) \\
& \widehat{S}(t)=B^{T} X \widehat{x}(t)=\left(\begin{array}{ccc}
0.0091 & 0.0038 & 0.0017 \\
-0.0090 & 0.0060 & 0.0145
\end{array}\right) \widehat{x}(t), \\
& u_{i}(t)=u_{\mathrm{eq} i}(t)+u_{c i}(t), \\
& u_{e i}(t)=\left(\rho_{i}(t, y)+\gamma_{1 i}\right) \operatorname{sgn}\left(S_{e}(t)\right) \text {, } \\
& u_{c i}(t)=-\left[\left(\rho_{i}(t, y)+\gamma_{1 i}\right)+\gamma_{2 i}\right] \operatorname{sgn}(\widehat{S}(t)) \text {, } \\
& u_{\mathrm{eq} i}(t)=-\left(B^{T} X B\right)^{-1} B^{T} X\left(A_{i} \widehat{x}(t)+A_{d i} \widehat{x}(t-d)\right) \text {, } \\
& u_{\mathrm{eq} 1}(t)=\left(\begin{array}{ccc}
2.000 & -3.0000 & -1.0000 \\
1.0000 & 2.1667 & 1.0000
\end{array}\right) \hat{x}(t) \\
& +\left(\begin{array}{ccc}
-0.3000 & 0.0000 & 0.1000 \\
0.1667 & -0.0667 & 0.1333
\end{array}\right) \widehat{x}(t-d), \\
& u_{\mathrm{eq} 2}(t)=\left(\begin{array}{ccc}
1.2000 & 0.5000 & -1.3000 \\
-1.4667 & -0.1333 & 1.3000
\end{array}\right) \hat{x}(t) \\
& +\left(\begin{array}{ccc}
-0.1000 & -0.1000 & -0.1000 \\
0.0267 & 0.0033 & -0.0000
\end{array}\right) \widehat{x}(t-d) .
\end{aligned}
$$

Let $\gamma_{11}=\gamma_{12}=60, \gamma_{21}=\gamma_{22}=40, \widehat{x}(t)=\left(\begin{array}{lll}0 & 0 & 0\end{array}\right)^{T}, t \epsilon$ $\left[\begin{array}{ll}-2,0 & 0\end{array}, \hat{x}(0)=\left(\begin{array}{lll}0 & 0 & 0\end{array}\right)^{T}\right.$. To restrain control signals from quiver, $\operatorname{sgn}(\widehat{S}(t))$ is replaced by $\widehat{S}(t) /(\|\widehat{S}(t)\|+0.01)$, and $S_{e}(t)$ is replaced by $S_{e}(t) /\left(\left\|S_{e}(t)\right\|+0.01\right) . \rho_{i}(t, y)$ is very tiny, compared with $\gamma_{1 i}$, so it is ignored.

Figures 1-4 testify the simulation results. Figures 1 and 2 show the time evolution of states and confirm asymptotic stability of the respective switched system. According to Figure 1, we know that the opened-loop switched system is instability and oscillation. Nevertheless, Figure 2 shows that the closed-loop switched system is stability. So it can be 


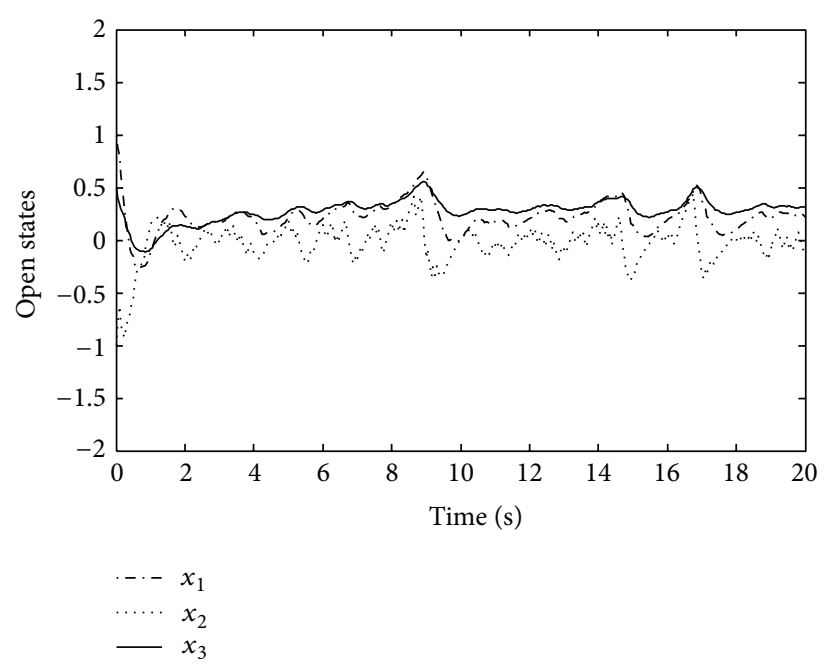

FIGURE 1: State response of the opened-loop switched system.

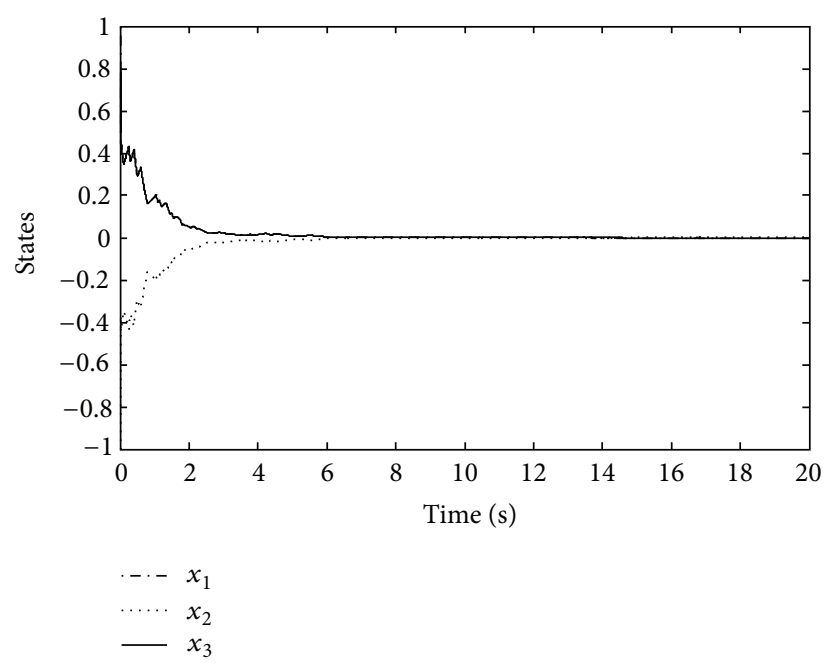

FIGURE 2: State response of the closed-loop switched system.

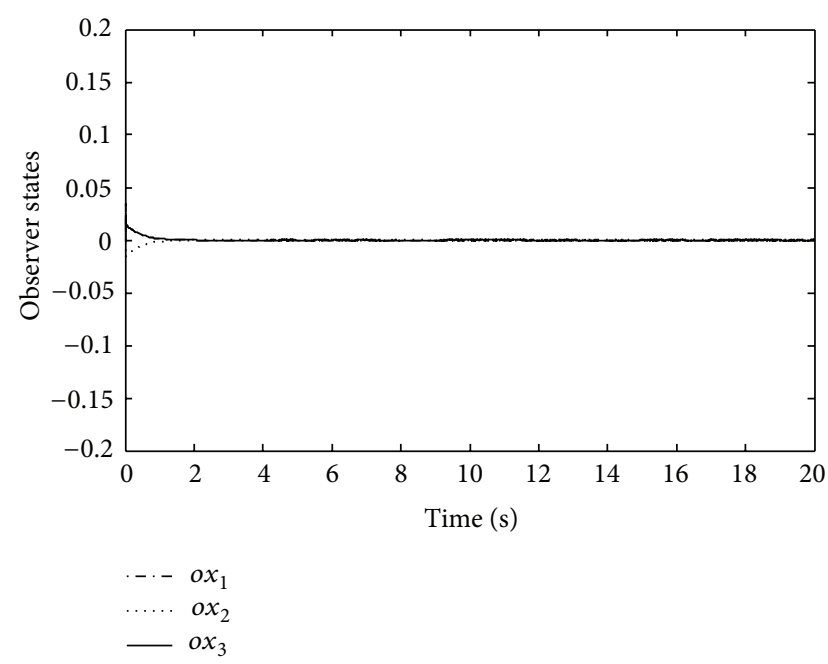

FIGURE 3: State response of the observer system.

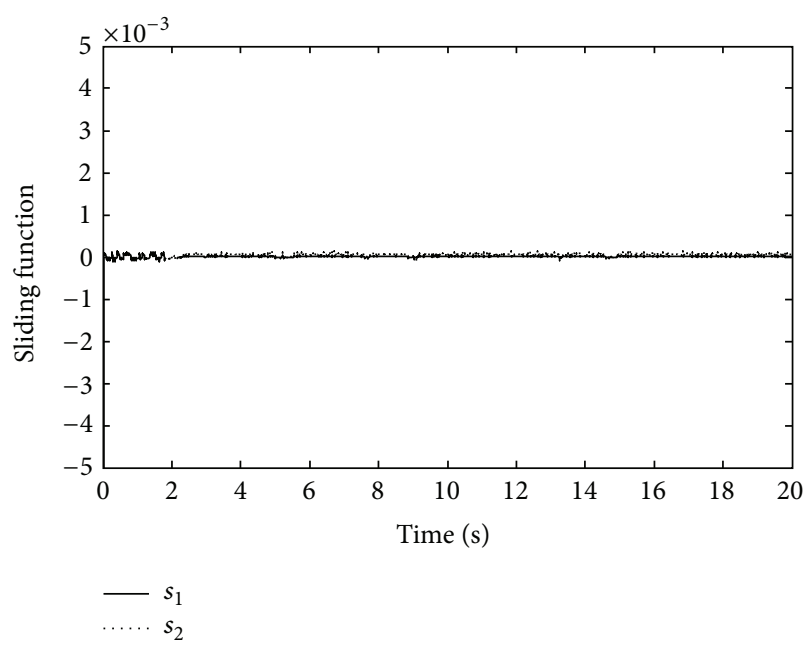

FIGURE 4: Sliding mode variables.

seen that state response of the closed-loop switched system is clearly superior to the opened-loop switched system.

And we check that, from Figure 3, the present observer based on sliding mode control scheme effectively eliminated effects of parameter uncertainties and nonlinearities and guaranteed asymptotic stability of the closed-loop system. Figure 4 shows that the sliding surface is accessibility but continue chattering. The chattering of the sliding mode variable $\widehat{S}(t)$ is treated as reduced-order compensation of dynamic system.

\section{Conclusion}

In this paper, an observer-based sliding mode control strategy is presented for a class of uncertain nonlinear state-delayed switched systems. Through this work, state-delayed switched system with immeasurable states and nonlinear uncertainties get better performance. Not only are the parameter uncertainties not needed to satisfy matching condition, but also the availabilities of all system states are no longer required. Meanwhile, a unique nonswitched sliding surface is designed in order to avoid repetitive jumps of the state trajectories between sliding surfaces leading to instability and chattering.

The weakness is that only the switching signal satisfies $\sigma(t)=i=\arg \left\{\min \left(V_{i}(t)\right)\right\}$, can the switched system be asymptotically stable. It is expected that the results developed in the paper can be extended to common cases that the underlying systems are involved in Markovian switching signal [25-27] or arbitrary switching signal.

\section{Acknowledgment}

This work is partially supported by the Natural Science Foundation of Heilongjiang Province (no. F201135).

\section{References}

[1] H. Lin and P. J. Antsaklis, "Switching stabilizability for continuous-time uncertain switched linear systems," IEEE 
Transactions on Automatic Control, vol. 52, no. 4, pp. 633-646, 2007.

[2] P. Varaiya, "Smart cars on smart roads: problems of control," IEEE Transactions on Automatic Control, vol. 38, no. 2, pp. 195207, 1993.

[3] D. Xie, N. Xu, and X. Chen, "Stabilisability and observerbased switched control design for switched linear systems," IET Control Theory \& Applications, vol. 2, no. 3, pp. 192-199, 2008.

[4] H. Dinh, R. Kamalapurkar, and S. Bhasin, "Dynamic neural network-based robust observers for second-order uncertain nonlinear systems," in Proceedings of the 50th IEEE Conference on Decision and Control and European Control, pp. 7543-7548, Orlando, Fla, USA, December 2011.

[5] W. Gao and J. C. Hung, "Variable structure control of nonlinear systems. A new approach," IEEE Transactions on Industrial Electronics, vol. 40, no. 1, pp. 45-55, 1993.

[6] Z. R. Xiang, C. Zhou, and R. H. Wang, "A LMI approach to observer design for a class of switched nonlinear systems," in Proceedings of the 27th Chinese Control Conference (CCC '08), pp. 285-288, Kunming, China, July 2008.

[7] Y. Niu, J. Lam, X. Wang, and D. W. C. Ho, "Observer-based sliding mode control for nonlinear state-delayed systems," International Journal of Systems Science, vol. 35, no. 2, pp. 139150, 2004.

[8] Y. Q. Xia and Y. M. Jia, "Robust sliding-mode control for uncertain time-delay systems: an LMI approach," IEEE Transactions on Automatic Control, vol. 48, no. 6, pp. 1086-1092, 2003.

[9] N. Sharma, S. Bhasin, Q. Wang, and W. E. Dixon, "RISE-based adaptive control of a control affine uncertain nonlinear system with unknown state delays," IEEE Transactions on Automatic Control, vol. 57, no. 1, pp. 255-259, 2012.

[10] Y. Niu, J. Lam, and X. Wang, "Sliding-mode control for uncertain neutral delay systems," IEEE Proceedings Part D, vol. 151, no. 1, pp. 38-44, 2004.

[11] L. Wu and W. X. Zheng, "Passivity-based sliding mode control of uncertain singular time-delay systems," Automatica, vol. 45, no. 9, pp. 2120-2127, 2009.

[12] J. H. Zhang, P. Shi, and Y. Q. Xia, "Robust adaptive sliding-mode control for fuzzy systems with mismatched uncertainties," IEEE Transactions on Fuzzy Systems, vol. 18, no. 4, pp. 700-711, 2010.

[13] M. Liu, X. Cao, and P. Shi, "Fault estimation and tolerant control for T-S fuzzy stochastic systems," IEEE Transactions on Fuzzy Systems, vol. PP, no. 99, 1 pages.

[14] P. Shi, Y. Xia, G. P. Liu, and D. Rees, "On designing of slidingmode control for stochastic jump systems," IEEE Transactions on Automatic Control, vol. 51, no. 1, pp. 97-103, 2006.

[15] L. Wu and D. W. C. Ho, "Sliding mode control of singular stochastic hybrid systems," Automatica, vol. 46, no. 4, pp. 779783, 2010.

[16] L. Wu, D. W. C. Ho, and C. W. Li, "Sliding mode control of switched hybrid systems with stochastic perturbation," Systems \& Control Letters, vol. 60, no. 8, pp. 531-539, 2011.

[17] L. Wu, X. Su, and P. Shi, "Sliding mode control with bounded $L_{2}$ gain performance of Markovian jump singular time-delay systems," Automatica, vol. 48, no. 8, pp. 1929-1933, 2012.

[18] L. Wu, W. X. Zheng, and H. Gao, "Dissipativity-based sliding mode control of switched stochastic systems," IEEE Transactions on Automatic Control, vol. 58, no. 3, pp. 785-791, 2012.

[19] L. Wu, C. H. Wang, and Q. Zeng, "Observer-based sliding mode control for a class of uncertain nonlinear neutral delay systems," Journal of the Franklin Institute, vol. 345, no. 3, pp. 233-253, 2008.
[20] Z. He, J. Wu, and G. Sun, "State estimation and sliding mode control of uncertain switched hybrid systems," International Journal of Innovative Computing, vol. 8, no. 10, pp. 7143-7156, 2012.

[21] I. R. Petersen, "A stabilization algorithm for a class of uncertain linear systems," Systems \& Control Letters, vol. 8, no. 4, pp. 351357, 1987.

[22] T. Iwasaki and R. E. Skelton, "All controllers for the general $H_{\infty}$ control problem: LMI existence conditions and state space formulas," Automatica, vol. 30, no. 8, pp. 1307-1317, 1994.

[23] H. H. Choi, "Variable structure control of dynamical systems with mismatched norm-bounded uncertainties: an LMI approach," Inernational Journal of Control, vol. 74, no. 13, pp. 1324-1334, 2001.

[24] C. Edwards, A. Akoachere, and S. K. Spurgeon, "Slidingmode output feedback controller design using linear matrix inequalities," IEEE Transactions on Automatic Control, vol. 46, no. 1, pp. 115-119, 2001.

[25] L. Wu, P. Shi, and H. Gao, "State estimation and slidingmode control of Markovian jump singular systems," IEEE Transactions on Automatic Control, vol. 55, no. 5, pp. 1213-1219, 2010.

[26] M. Liu, P. Shi, L. Zhang, and X. Zhao, "Fault-tolerant control for nonlinear Markovian jump systems via proportional and derivative sliding mode observer technique," IEEE Transactions on Circuits and Systems I, vol. 58, no. 11, pp. 2755-2764, 2011.

[27] L. Ma, F. Da, and K.-J. Zhang, "Exponential $H_{\infty}$ filter design for discrete time-delay stochastic systems with Markovian jump parameters and missing measurements," IEEE Transactions on Circuits and Systems I, vol. 58, no. 5, pp. 994-1007, 2011. 


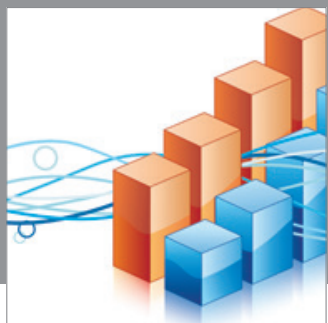

Advances in

Operations Research

mansans

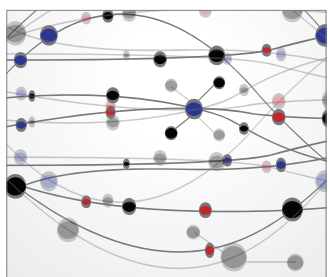

The Scientific World Journal
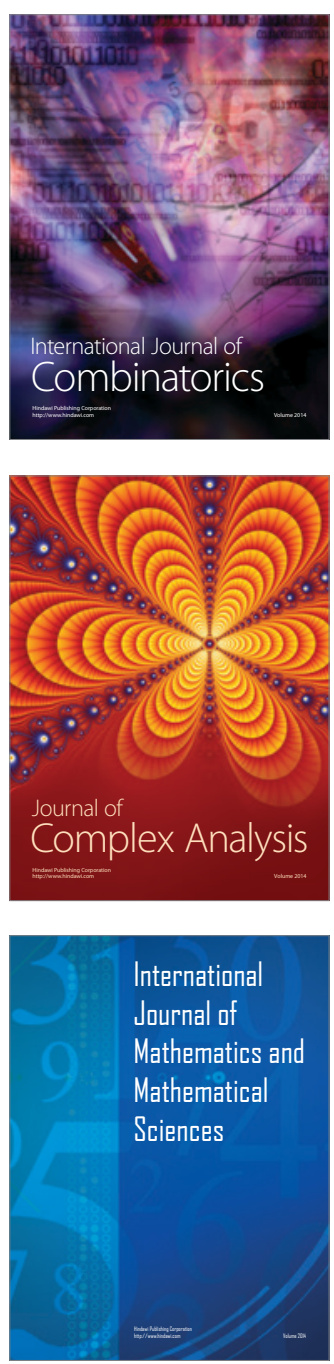


Submit your manuscripts at http://www.hindawi.com
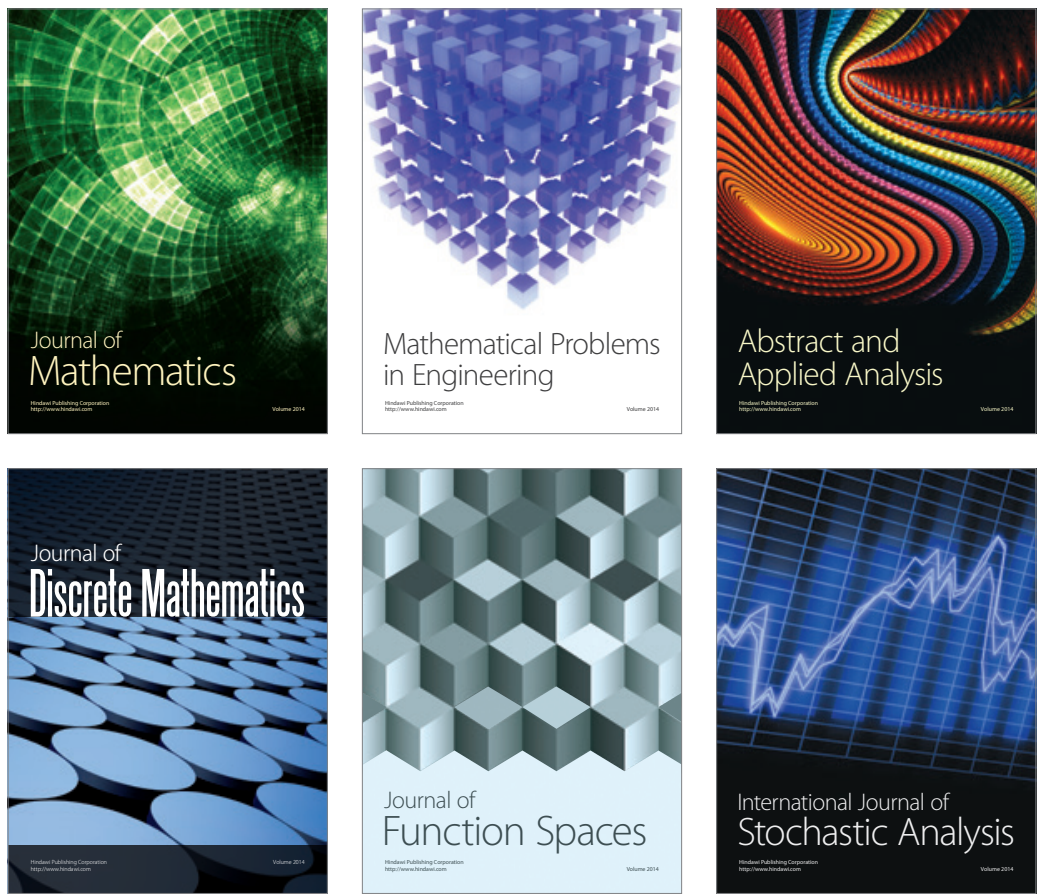

Journal of

Function Spaces




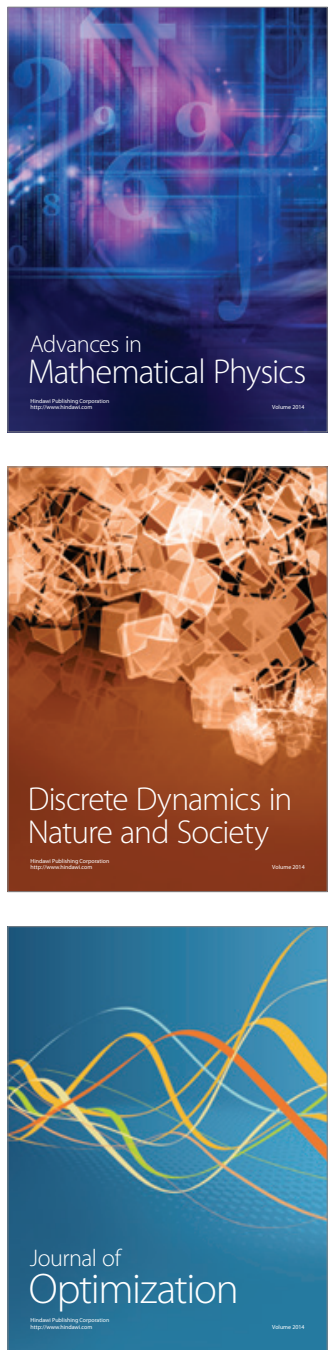